\title{
FINITELY GENERATED EXTENSIONS OF DIFFERENCE FIELDS
}

\author{
RICHARD M. COHN
}

Let $\mathcal{F}, \mathfrak{F}, K$ be difference fields such that $\mathcal{F} \subseteq \mathfrak{H} \subseteq K$. We shall prove that if $K$ is a finitely generated extension of $\mathcal{F}, K=\mathcal{F}\left\langle\alpha_{1}, \alpha_{2}, \cdots\right.$, $\left.\alpha_{n}\right\rangle,{ }^{1}$ then $\mathcal{H C}$ is also a finitely generated extension of $\mathcal{F}$.

We introduce a new notation for the $\alpha_{i}$. Let $\beta_{1}, \cdots, \beta_{q}$ denote a subset of the $\alpha_{i}$ annulling no nonzero difference polynomial with coefficients in $\mathcal{H C}$ and such that each $\alpha_{i}$ annuls some nonzero difference polynomial with coefficients in $\mathscr{T}\left\langle\beta_{1}, \cdots, \beta_{q}\right\rangle$. We denote the $\alpha_{i}$ not included among the $\beta_{i}$ by $\gamma_{1}, \cdots, \gamma_{p}, p=n-q$.

Let $\Lambda$ be the reflexive prime difference ideal in $\mathcal{H C}\left\{u_{1}, \ldots, u_{q}\right.$; $\left.y_{1}, \cdots, y_{p}\right\}$ with the generic zero $u_{i}=\beta_{i}, i=1, \cdots, q ; y_{j}=\gamma_{j}$, $j=1, \cdots, p$. We denote a characteristic set of $\Lambda$ by

$$
A_{10}, \cdots, A_{1 k_{1}} ; A_{20}, \cdots, A_{2 k_{2}} ; \cdots ; A_{p 0}, \cdots, A_{p k_{p}},
$$

where $A_{i 0}$ introduces ${ }^{2} y_{i}$. Let $G$ be the difference field formed by adjoining the coefficients ${ }^{3}$ of the $A_{i j}$ to $\mathcal{F}$. Evidently $G \subseteq \mathcal{H}$. The result stated above will follow when we show that $G=\mathfrak{F}$.

We shall describe what we mean by the characteristic sequences $B_{i j}, i=1, \cdots, p ; j=0,1, \cdots$, of $\Lambda$ formed from (1). This concept has been previously defined only in special cases.

Let $t_{i}$ denote the order of $A_{i 0}$ in $y_{i}$. We let $B_{10}=A_{10}$. Suppose $B_{10}, \cdots, B_{1, k-1}$ have been defined. Then, if there is an $A_{1 j}$ of order $t_{1}+k$ in $y_{1}$, we let $B_{1 k}$ be that $A_{1 j}$. Otherwise $B_{1 k}$ is defined as the remainder ${ }^{4}$ of the transform of $B_{1, k-1}$ with respect to the chain $B_{10}, \cdots$, $B_{1, k-1}$. It is easy to see that, for any $r, B_{1 r}$ is of order $t_{1}+r$ in $y_{1}$ and, unless it is equal to some $A_{1 j}$, of the same degree in the $\left(t_{1}+r\right)$ th transform of $y_{1}$ as is $B_{1, r-1}$ in the $\left(t_{1}-1+r\right)$ th transform of $y_{1}$.

Let $B_{20}=A_{20}$. Suppose $B_{20}, \cdots, B_{2, k-1}$ have been defined. Then if

Received by the editors March 1, 1954.

1 The brackets \langle\rangle denote field adjunction of the enclosed elements and their transforms so as to form a difference field. Similarly, brackets \{ \} denote ring adjunction of the enclosed elements and their transforms. Field and ring adjunctions in the usual sense are denoted by brackets ( ) and [ ] respectively. For other terms used see [1] (where the term "basic set" corresponds to our "characteristic set") and [3].

2 If $p=0, \Lambda$ is the ideal consisting only of 0 , and no $A_{i j}$ are defined.

${ }^{3}$ If $p=0$, we define $G$ to be $\mathcal{F}$.

4 Throughout this discussion we form the remainder treating the $B_{i j}$ not as difference polynomials but as polynomials as in Chapter IV of [2]. The $y_{i j}, j \geqq t_{i}$, are ordered lexicographically. The remaining $y_{i j}$ and the $u_{i j}$ precede them and are ordered among themselves in any convenient way. Of course, only a finite number of indeterminates are present and need be ordered at any step. 
there is an $A_{2 j}$ of order $t_{2}+k$ we let $B_{2 k}$ be that $A_{2 j}$. Otherwise $B_{2 k}$ is defined as the remainder of the transform of $B_{2, k-1}$ with respect to the chain $B_{10}, \cdots, B_{1 r} ; B_{20}, \cdots, B_{2, k-1}$, where $r$ is chosen as the least integer such that no transform of $y_{10}$ occurring in $B_{20}, \cdots, B_{2, k-1}$ or the transform of $B_{2, k-1}$ is of order exceeding $t_{1}+r$. Proceeding similarly we let $B_{30}=A_{30}$. When $B_{30}, \cdots, B_{3, k-1}$ have been defined, we define $B_{3 k}$ as the $A_{3 j}$ of the proper order, if such exists, or as the remainder of the transform of $B_{3, k-1}$ with respect to $B_{10}, \cdots, B_{18}$; $B_{20}, \cdots, B_{2 r}$, where $s$ and $r$ are such that no transform of $y_{20}$ occurring in $B_{30}, \cdots, B_{3, k-1}$ or the transform of $B_{3, k-1}$ is of order exceeding $t_{2}+r$, and that no transform of $y_{10}$ occurring in these polynomials or in $B_{20}, \cdots, B_{2 r}$ is of order exceeding $t_{1}+s$.

Continuing in this way we define the $B_{i j}, i=1, \cdots, p ; j=0,1, \cdots$. Each $B_{i j}$ is of order $t_{i}+j$ in $y_{i}$, and it is either a polynomial of the characteristic set of $\Lambda$ which is of this order in $y_{i}$ and free of $y_{k}, k>i$, or it is of the same degree in the $\left(t_{i}+j\right)$ th transform of $y_{i}$ as is $B_{i, j-1}$ in the $\left(t_{i}-1+j\right)$ th transform of $y_{i}$. Of course, $B_{i j}$ is free of $y_{k l}, k>i$.

Given an integer $r \geqq 0$ we let $s_{p}$ denote the maximum of $t_{p}$ and $r$. Let $r_{p}=s_{p}-t_{p}$. We then define $s_{p-1}$ to be the maximum of $t_{p-1}, r$, and the order of the highest transform of $y_{p-1}$ appearing in the polynomials $B_{p 0}, \cdots, B_{p r_{p}}$, and let $r_{p-1}=s_{p-1}-t_{p-1}$. We define $s_{p-2}$ as the maximum of $t_{p-2}, r$, and the order of the highest transform of $y_{p-2}$ occurring in $B_{p-1,0}, \cdots, B_{p-1, r_{p-1}} ; B_{p 0}, \cdots, B_{p r_{p}}$. Continuing in this way we define successively $s_{p-3}, s_{p-4}, \cdots, s_{1}$ and let $r_{i}=s_{i}-t_{i}$, $i=1, \cdots, p$. Then

$$
B_{10}, \cdots, \quad B_{1 r_{1}} ; \quad B_{20}, \cdots, B_{2 r_{2}} ; \cdots ; B_{p 0}, \cdots, B_{p r_{p}}
$$

is a chain. For $s$ such that no $u_{i j}, j>s$, occurs in (2) we define $\Lambda_{s r}$ as the prime p. i. (polynomial ideal ${ }^{5}$ ) in the indeterminates $u_{i j}$, $i=1, \cdots, q ; j=0,1, \cdots, s$, and $y_{k m}, k=1, \cdots, p ; m=0,1, \cdots, s_{k}$, which consists of those polynomials of $\Lambda$ which involve only these $u_{i j}$ and $y_{k m}$. Then (2) constitutes a characteristic set for $\Lambda_{s r}$ with $B_{i j}$ introducing $y_{i, t_{i}+j}$. The parametric indeterminates of $\Lambda_{s r}$ corresponding to this choice of characteristic set are those $u_{i j}$ occurring among its indeterminates and the $y_{k m}$ with $m<t_{m}$. We note that all coefficients of the $B_{i j}$ are rational combinations of the coefficients appearing in (1) and their transforms.

Let $\lambda$ be any element of $\mathcal{H}$. It will evidently suffice to show that $\lambda$ is in $G$. We choose a positive integer $r$ such that $\lambda$ is in the field formed by adjoining to $\mathcal{F}$ the $\alpha_{i j}, i=1, \cdots, n ; j=0, \cdots, r$. Let $s \geqq r$ be such that, with the $r$ just chosen, (2) is a characteristic set of

${ }^{6}$ Following Chapter IV of [2] we use this term to distinguish ideals of polynomials in the usual sense from difference ideals. 
a prime p. i. $\Lambda_{s r}$. By the last remark of the preceding paragraph the coefficients of (2) are in $G$. Since also $G \subseteq \mathcal{K C}$ it is readily seen that (2) is the characteristic set of a prime p. i. II with coefficients in $G$ and involving the same indeterminates as $\Lambda_{s r}$. Similarly (2) is the characteristic set of a prime p. i. $\Pi^{\prime}$ with coefficients in $G(\lambda)$ and involving the same indeterminates as $\Lambda_{s r}$.

We obtain a generic zero of $\Lambda_{s r}, \Pi$, or $\Pi^{\prime}$ by putting $u_{i j}=\beta_{i j}$; $y_{i j}=\gamma_{i j}$ for the appropriate ranges of the subscripts. We shall denote by $\delta_{k}$, where $k$ ranges over a suitable set of integers, those $\beta_{i j}$ and $\gamma_{i j}$ of the generic zero which have been equated to the $u_{i j}$ and $y_{i j}$ of the previously described set of parametric indeterminates of $\Lambda_{s r}$ (which are also, of course, a set of parametric indeterminates for either $\Pi$ or $\left.\Pi^{\prime}\right)$. The remaining $\gamma_{i j}$ of the generic zero shall henceforth be denoted by $\epsilon_{m}$, where $m$ ranges over a suitable set of integers.

The degree of $G\left(\delta_{k}, \epsilon_{m}\right)$ with respect to $G\left(\delta_{k}\right)$ is given by the product of the degrees ${ }^{6}$ of the polynomials of (2) in the indeterminates of $\Pi$ which they respectively introduce. We see in the same way that this product is the degree of $G(\lambda)\left(\delta_{k}, \epsilon_{m}\right)$ with respect to $G(\lambda)\left(\delta_{k}\right)$. But the fields $G(\lambda)\left(\delta_{k}, \epsilon_{m}\right)$ and $G\left(\delta_{k}, \epsilon_{m}\right)$ coincide because, by the stipulations concerning $r$ and $s, \lambda$ is in $G\left(\delta_{k}, \epsilon_{m}\right)$. Hence the degrees of $G\left(\delta_{k}, \epsilon_{m}\right)$ with respect to its two subfields $G\left(\delta_{k}\right)$ and $G\left(\lambda, \delta_{k}\right)$ are equal. Since these degrees are finite it follows that these subfields must be identical. In other words, $\lambda$ is in $G\left(\delta_{k}\right)$.

We thus see that there exist elements $P$ and $Q$ in $G\left[\delta_{k}\right]$, with $P$ not equal to zero, such that $P \lambda=Q$. Now the $\delta_{k}$ annul no nonzero polynomial with coefficients in $G(\lambda)$. Hence the relation $P \lambda=Q$ must be an identity in the $\delta_{k}$. By equating coefficients of a suitable power product of the $\delta_{k}$ on both sides of this equation we find $p \lambda=q, p$ and $q$ in $G$, and $p \neq 0$. Hence $\lambda$ is in $G$. This completes the proof.

\section{REFERENCES}

1. J. L. Doob and J. F. Ritt, Systems of algebraic difference equations, Amer. J. Math. vol. 55 (1933) pp. 505-514.

2. J. F. Ritt, Differential algebra, Amer. Math. Soc. Colloquium Publications, vol. $33,1950$.

3. R. M. Cohn, Extensions of difference fields, Amer. J. Math. vol. 74 (1952) pp. 507-530.

\section{RUTGERS UNIVERSITY}

6 This follows from the work on pp. 89 and 90 of [2]. The inductive argument given there shows that a generic zero of $I I$ can be constructed by transcendental adjunctions followed by successive algebraic adjunctions of degrees equal to the degrees of the polynomials of the characteristic set in the indeterminates they introduce. 